\title{
A COMBINATORIAL PROOF OF A PARTITION IDENTITY OF ANDREWS AND STANLEY
}

\author{
ANDREW V. SILLS \\ Received 26 January 2004 and in revised form 5 February 2004

\begin{abstract}
In his paper, "On a partition function of Richard Stanley," George Andrews proves a certain partition identity analytically and asks for a combinatorial proof. This paper provides the requested combinatorial proof.
\end{abstract}

2000 Mathematics Subject Classification: 05A17.

1. Introduction. In [6], Stanley posed a problem on partitions. In [2], Andrews studies the function $S(r, s ; n)$ which equals the number of partitions $\pi$ of $n$ such that $\pi$ has $r$ odd parts and the conjugate $\pi^{\prime}$ of $\pi$ has $s$ odd parts, and proves a result from which he solves Stanley's problem as a corollary.

Andrews also states some additional interesting corollaries, including the following theorem.

\section{THEOREM 1.1.}

$$
\sum_{n, r, s \geqq 0} S(r, s ; n) z^{r} y^{s} q^{n}=\prod_{j=1}^{\infty} \frac{\left(1+y z q^{2 j-1}\right)}{\left(1-q^{4 j}\right)\left(1-z^{2} q^{4 j-2}\right)\left(1-y^{2} q^{4 j-2}\right)} .
$$

He proves this theorem analytically as the limiting case of a certain polynomial identity [2, Theorem 1]. At the end of the paper, Andrews states that (1.1) "cries out for a combinatorial proof." The purpose of this paper is to present such a proof.

2. Definitions and notation. The following definitions and notations follow Macdonald [4].

A partition $\pi$ of an integer $n$ is a nonincreasing sequence of nonnegative integers containing only finitely many nonzero terms such that the sum of the terms is $n$. Thus,

$$
\pi=\left(\pi_{1}, \pi_{2}, \pi_{3}, \ldots\right)
$$

with

$$
\begin{gathered}
\pi_{1} \geqq \pi_{2} \geqq \pi_{3} \geqq \cdots \geqq 0, \\
\sum_{i=1}^{\infty} \pi_{i}=n,
\end{gathered}
$$


and $\pi_{i} \in \mathbb{Z}$. Since the tail of such a sequence must, by definition, be an infinite string of zeros, it will be convenient to suppress this in the notation. Thus the partition $(2,2,1,1,1,0,0,0,0,0, \ldots)$ is normally written as $(2,2,1,1,1)$. In any event, no distinction will be drawn among sequences written with or without any number of trailing zeros.

The nonzero terms $\pi_{i}$ in (2.1) are called the parts of the partition $\pi$. The number of parts of $\pi$ is called the length of $\pi$ and is denoted $l(\pi)$. Additionally, I will follow Stanley [6] and Andrews [2] and let $\mathcal{O}(\pi)$ denote the number of odd parts in the partition $\pi$.

The set of all partitions is denoted $\mathscr{P}$.

An alternate notation for a partition $\pi$ is

$$
\pi=\left\langle 1^{m_{1}} 2^{m_{2}} \cdots r^{m_{r}} \cdots\right\rangle
$$

which means that exactly $m_{i}$ of the parts of $\pi$ are equal to $i$. The number $m_{i}=m_{i}(\pi)$ is called the multiplicity of $i$ in $\pi$. Thus,

$$
(6,5,5,3,3,3,3,2)=\left\langle 23^{4} 5^{2} 6\right\rangle .
$$

If $\pi=\left(\pi_{1}, \pi_{2}, \pi_{3}, \ldots\right)$ and $\lambda=\left(\lambda_{1}, \lambda_{2}, \lambda_{3}, \ldots\right)$ are two partitions, their sum is defined termwise:

$$
(\pi+\lambda)_{i}:=\pi_{i}+\lambda_{i}
$$

for $i \in \mathbb{Z}_{+}$.

The union of two partitions $\lambda$ and $\pi$ is obtained by merging the entries of $\lambda$ with those of $\pi$ and arranging the resulting entries in nonincreasing order, for example,

$$
(3,3,2,1) \cup(5,3,2,2)=(5,3,3,3,2,2,2,1)
$$

The conjugate of a partition $\pi=\left(\pi_{1}, \pi_{2}, \pi_{3}, \ldots\right)$, denoted $\pi^{\prime}=\left(\pi_{1}^{\prime}, \pi_{2}^{\prime}, \pi_{3}^{\prime}, \ldots\right)$, is the partition such that

$$
\pi_{i}^{\prime}=\sum_{j \geqq i} m_{j}(\pi)
$$

Note that $\pi^{\prime \prime}=\pi, \pi_{1}=l\left(\pi^{\prime}\right)$, and

$$
m_{i}\left(\pi^{\prime}\right)=\pi_{i}-\pi_{i+1}
$$

Usually, the conjugate of a partition is thought of in terms of the transposition of its Ferrers graph or Young diagram; see, for example, [1, page 6] or [4, page 2].

Finally, define the parity function

$$
P(i)= \begin{cases}0 & \text { if } i \text { is even } \\ 1 & \text { if } i \text { is odd }\end{cases}
$$




\section{Combinatorial proof of Theorem 1.1}

3.1. The plan. I will start with the set of all partitions $\mathscr{P}$ counted in such a way so that it is easily seen to be generated by the infinite product on the right-hand side of (1.1). I will then map $\mathscr{P}$ bijectively onto itself while keeping track of enough of the internal counting to see that (1.1) holds.

In order to keep the presentation as straightforward as possible, the mapping will be presented in three simple steps (the maps $\alpha, \beta$, and $\gamma$ ), and the bijection will be the composition of these three maps.

3.2. The generating function. By standard elementary reasoning (see [1, Chapters 1 and 2]),

$$
\prod_{j=1}^{\infty} \frac{1}{1-q^{4 j}}
$$

is the generating function for partitions into parts $\equiv 0(\bmod 4)$,

$$
\prod_{j=1}^{\infty} \frac{1}{1-z^{2} q^{4 j-2}}
$$

is the generating function for partitions into parts $\equiv 2(\bmod 4)$, where each part is counted with a weight of $z^{2}$, and

$$
\prod_{j=1}^{\infty} \frac{1+y x q^{2 j-1}}{1-y^{2} q^{4 j-2}}
$$

is the generating function for partitions into odd parts, where each part is counted with weight $y$ and each distinct integer of odd multiplicity is counted with weight $x$.

Thus,

$$
\sum_{n \geqq 0} R(t, \rho, s ; n) x^{t} z^{2 \rho} y^{s} q^{n}=\prod_{j=1}^{\infty} \frac{\left(1+y x q^{2 j-1}\right)}{\left(1-q^{4 j}\right)\left(1-z^{2} q^{4 j-2}\right)\left(1-y^{2} q^{4 j-2}\right)},
$$

where $R(n)$ is the number of partitions of $n$ with $s$ odd parts, $\rho$ parts congruent to $2(\bmod 4)$, and $t$ different odd integers of odd multiplicity.

Upon setting $x$ equal to $z$ and renaming $2 \rho+t$ as $r$, we see that the infinite product of (1.1) generates partitions with exactly $s$ odd parts and such that twice the number of parts congruent to $2(\bmod 4)$ plus the number of different odd integers of odd multiplicity is exactly $r$. Thus, by mapping each of these partitions to a partition with exactly $r$ odd parts and $s$ odd parts in its conjugate, we will have a bijective proof that (1.1) holds. 
3.3. The mapping. Start with an arbitrary partition $\kappa=\left\langle 1^{m_{1}(\kappa)} 2^{m_{2}(\kappa)} 3^{m_{3}(\kappa)} \cdots\right\rangle$. Define a map

$$
\alpha: \mathscr{P} \longrightarrow \mathscr{A} \times \mathscr{B}
$$

where $\mathscr{A}$ is the set of all partitions with no repeated odd parts and $\mathscr{B}=\mathscr{P} \backslash \mathscr{A}$ is the set of partitions with only odd parts of even multiplicity, by

$$
\alpha(\kappa)=(\lambda, \mu)
$$

where

$$
\begin{aligned}
& \lambda=\left\langle 1^{P\left(m_{1}(\kappa)\right)} 2^{m_{2}(\kappa)} 3^{P\left(m_{3}(\kappa)\right)} 4^{m_{4}(\kappa)} \cdots\right\rangle, \\
& \mu=\left\langle 1^{m_{1}(\kappa)-P\left(m_{1}(\kappa)\right)} 3^{m_{3}(\kappa)-P\left(m_{3}(\kappa)\right)} 5^{m_{5}(\kappa)-P\left(m_{5}(\kappa)\right)} \cdots\right\rangle .
\end{aligned}
$$

Informally, $\alpha$ separates $\kappa$ into two partitions $\lambda$ and $\mu$, where $\lambda$ gets all of the even parts of $\kappa$ and one copy of each odd part of odd multiplicity in $\kappa$, and $\mu$ gets what is left over, that is, even quantities of odd parts.

Notice that

$$
\mathcal{O}(\lambda)=t, \quad l(\mu)=\mathcal{O}(\mu)=s-t .
$$

Define the map $\beta_{1}: \mathscr{A} \rightarrow \mathscr{C}$ so that $\beta_{1}(\lambda):=\zeta=\left(\zeta_{1}, \zeta_{2}, \ldots\right)$, where for $i \in \mathbb{Z}_{+}$,

$$
\zeta_{2 i-1}=\left\lfloor\frac{\lambda_{i}+1}{2}\right\rfloor, \quad \zeta_{2 i}=\left\lfloor\frac{\lambda_{i}}{2}\right\rfloor,
$$

or equivalently,

$$
m_{i}(\zeta)=P\left(m_{2 i-1}(\kappa)\right)+2 m_{2 i}(\kappa)+P\left(m_{2 i+1}(\kappa)\right) .
$$

Informally, this means that every even part $2 j$ of $\lambda$ is mapped by $\beta_{1}$ to a pair of $j$ 's and each and every odd part $2 j+1$ is mapped to the pair $j+1, j$. So, $\mathscr{C}$ is the set of partitions $\zeta=\left(\zeta_{1}, \zeta_{2}, \ldots\right)$ such that for all $i \in \mathbb{Z}_{+}$,

$$
\zeta_{2 i-1}-\zeta_{2 i} \leqq 1
$$

Define the map $\beta_{2}: \mathscr{B} \rightarrow \mathscr{D}$ so that $\xi:=\beta_{2}(\mu)=\mu^{\prime}$.

Note that $\mathscr{D}$ is the set of partitions $\xi$ such that for all $i \in \mathbb{Z}_{+}, \xi_{2 i}=\xi_{2 i+1}$, and $\xi_{i}$ is even. Also, $\mathscr{O}(\zeta)=2 \rho+t, \mathcal{O}(\xi)=0$, and $\mathscr{O}\left(\xi^{\prime}\right)=\mathscr{O}(\mu)=s-t$.

Claim 3.1. $O\left(\zeta^{\prime}\right)=t$.

Proof of Claim 3.1. To see this, notice that by (3.11), the only way that $\zeta_{1}^{\prime}$ can be odd is if 1 is a part of $\lambda$ (which of course means that $m_{1}(\kappa)$ is odd). This is so because every part of $\lambda$ other than $a 1$ is mapped to a pair of parts, but 1 is mapped to $(1,0)$, which by definition is only one part since 0 does not count as a part.

Next, the only way that $\zeta_{2}^{\prime}$ can be odd is if $\lambda$ contains a 3 ; and in general, the only way that $\zeta_{i}^{\prime}$ can be odd is if $\lambda$ contains $2 i-1$ as a part (which means that $m_{2 i-1}(\kappa)$ is odd). 
Finally, define a map $\gamma: \mathscr{C} \times \mathscr{D} \rightarrow \mathscr{P}$ by $\pi=\gamma(\zeta, \xi):=\zeta+\pi$. Thus, we have $\mathscr{O}(\pi)=$ $\mathcal{O}(\zeta)+\mathcal{O}(\xi)=2 \rho+t=: r$.

Claim 3.2. $\mathcal{O}\left(\pi^{\prime}\right)=\mathscr{O}\left(\zeta^{\prime}\right)+\mathscr{O}\left(\xi^{\prime}\right)=s$ as desired, that is, no odd parts in the conjugate are "lost" through the addition of the partitions $\zeta$ and $\xi$.

Proof OF ClAim 3.2.

$$
\begin{aligned}
\mathcal{O}\left(\pi^{\prime}\right) & =\sum_{i \geqq 1} m_{2 i-1}\left(\pi^{\prime}\right) \\
& =\sum_{i \geqq 1}\left(\pi_{2 i-1}-\pi_{2 i}\right) \\
& =\sum_{i \geqq 1}\left(\left(\zeta_{2 i-1}+\xi_{2 i-1}\right)-\left(\zeta_{2 i}+\xi_{2 i}\right)\right) \\
& =\sum_{i \geqq 1}\left(\left(\left\lfloor\frac{\lambda_{i}+1}{2}\right\rfloor+\mu_{2 i-1}^{\prime}\right)-\left(\left\lfloor\frac{\lambda_{i}}{2}\right\rfloor+\mu_{2 i}^{\prime}\right)\right) \\
& =\sum_{i \geqq 1}\left(\left\lfloor\frac{\lambda_{i}+1}{2}\right\rfloor-\left\lfloor\frac{\lambda_{i}}{2}\right\rfloor+\mu_{2 i-1}^{\prime}-\mu_{2 i}^{\prime}\right) \\
& =\sum_{i \geqq 1}\left\lfloor\frac{\lambda_{i}+1}{2}\right\rfloor-\left\lfloor\frac{\lambda_{i}}{2}\right\rfloor+m_{2 i-1}(\mu) \quad \text { by }(2.8) \\
& =\mathbb{O}(\lambda)+\mathbb{O}(\mu) \\
& =t+(s-t) \\
& =s
\end{aligned}
$$

where the third to last equality follows from the fact that $\left\lfloor\left(\lambda_{i}+1\right) / 2\right\rfloor-\left\lfloor\lambda_{i} / 2\right\rfloor$ equals 0 if $i$ is even, and equals 1 if $i$ is odd.

Now we consider the invertibility of each of the maps. It is easy to see that $\alpha$ is invertible; $\alpha^{-1}(\lambda, \mu)=\lambda \cup \mu$. $\beta_{2}^{-1}=\beta_{2}$ since conjugation is an involution. $\beta_{1}^{-1}(\zeta)=\lambda=$ $\left(\lambda_{1}, \lambda_{2}, \ldots\right)$, where

$$
\lambda_{i}=\zeta_{2 i-1}+\zeta_{2 i}
$$

for $i \in \mathbb{Z}_{+}$.

The invertibility of $\gamma$, however, is more subtle. Given any partition $\pi$, we need to "split it" into the sum of two partitions $\zeta \in \mathscr{C}$ and $\xi \in \mathscr{D}$. It is sufficient to recover $\xi_{1}$ and $\xi_{2 i}$ for $i \geqq 1$ since $\xi_{2 i}=\xi_{2 i+1}$ for all $i \geqq 1$, and once we have $\xi$, we know $\zeta$ since $\zeta+\xi=\pi$. Of course, we need to do this given only the partition $\pi$.

The easiest case is finding $\xi_{1}$ since we know that $\xi_{1}=s-t$. Both $s$ and $t$ are readily available to us in $\pi$ : besides counting the number of odd parts in $\kappa, s$ counts the number of odd parts in $\pi^{\prime}$. Now $t$ counts the number of odd parts of odd multiplicity in $\kappa$ which translates into the number of odd parts in $\lambda$, which translates into the number of pairs $\left(\zeta_{2 i-1}, \zeta_{2 i}\right)$ in $\zeta$ whose sum is odd, which in turn translates to the number of pairs $\left(\pi_{2 i-1}, \pi_{2 i}\right)$ in $\pi$ whose sum is odd (since all parts of $\xi$ are even). Thus we can recover $\xi_{1}$ from $\pi$. 
Now that we have both $\pi$ and $\xi_{1}$ in hand, we can recover the rest of $\xi$.

Claim 3.3. For $i \geqq 1$,

$$
\xi_{2 i}=\xi_{1}-\sum_{j=1}^{i}\left(\pi_{2 i-1}-\pi_{2 i}\right)+\sum_{j=1}^{i} P\left(\pi_{2 i-1}+\pi_{2 i}\right) .
$$

\section{Proof OF Claim 3.3.}

$$
\begin{aligned}
\xi_{1}- & \sum_{j=1}^{i}\left(\pi_{2 j-1}-\pi_{2 j}\right)+\sum_{j=1}^{i} P\left(\pi_{2 j-1}+\pi_{2 j}\right) \\
& =\xi_{1}-\sum_{j=1}^{i}\left(\zeta_{2 j-1}+\xi_{2 j-1}-\zeta_{2 j}-\xi_{2 j}\right)+\sum_{j=1}^{i} P\left(\lambda_{j}\right) \\
& =\xi_{1}-\sum_{j=1}^{i}\left(\left\lfloor\frac{\lambda_{j}+1}{2}\right\rfloor+\xi_{2 j-1}-\left\lfloor\frac{\lambda_{j}}{2}\right\rfloor-\xi_{2 j}\right)+\sum_{j=1}^{i} P\left(\lambda_{j}\right) \\
& =\xi_{1}+\sum_{j=1}^{i}\left(P\left(\lambda_{j}\right)-\left(\left\lfloor\frac{\lambda_{j}+1}{2}\right\rfloor-\left\lfloor\frac{\lambda_{j}}{2}\right\rfloor\right)\right)-\sum_{j=1}^{i}\left(\xi_{2 j-1}-\xi_{2 j}\right) \\
& =\xi_{1}+\sum_{j=1}^{i} 0-\left(\xi_{1}+\xi_{2}\right)-\cdots-\left(\xi_{2 i-1}-\xi_{2 i}\right) \\
& =\xi_{1}-\xi_{1}+\left(\xi_{2}-\xi_{3}\right)+\cdots+\left(\xi_{2 i-2}-\xi_{2 i-1}\right)+\xi_{2 i} \\
& =\xi_{2 i} .
\end{aligned}
$$

4. Example. Start with a partition

$$
\kappa=\left\langle 1^{5} 23^{3} 4^{2} 56^{2} 7^{2} 9\right\rangle
$$

Under $\alpha$, the parts of $\kappa$ are separated into $\lambda$ and $\mu$, where $\lambda$ gets all of the even parts of $\kappa$, and one copy of each odd part of odd multiplicity, and $\mu$ gets what is left over, that is, even quantities of odd parts:

$$
\begin{aligned}
(\lambda, \mu) & =\left(\left\langle 1234^{2} 56^{2} 9\right\rangle,\left\langle 1^{4} 3^{2} 7^{2}\right\rangle\right) \\
& =((9,6,6,5,4,4,3,2,1),(7,7,3,3,1,1,1,1)) .
\end{aligned}
$$

Next, $\beta_{1}$ takes each even part $2 j$ of $\lambda$ to a pair of $j$ 's and each odd part $2 j+1$ to the pair $j+1, j$, and $\beta_{2}$ maps $\zeta$ to its conjugate:

$$
(\zeta, \xi)=((5,4,3,3,3,3,3,2,2,2,2,2,2,1,1,1,1,0),(8,4,4,2,2,2,2))
$$

Finally, $\gamma$ merges $\zeta$ and $\xi$ into a single partition $\pi$ via partition addition:

$$
\pi=(13,8,7,5,5,5,5,2,2,2,2,2,2,1,1,1,1)=\left\langle 1^{4} 2^{6} 5^{4} 7813\right\rangle .
$$


Now we consider the inverse map, pretending for the moment that we only know $\pi$. As in the general case, the hardest part will be to separate $\pi$ into $\zeta$ and $\xi$. First of all, $\pi^{\prime}=\left\langle 1^{5} 23^{2} 7^{3} 1317\right\rangle$, so $\mathcal{O}\left(\pi^{\prime}\right)=s=12$. Next, notice that $\pi=(13,8,7,5,5,5,5,2,2,2$, $2,2,2,1,1,1,1,0)$ has $t=4$ pairs of opposite parity. Thus we know that $\xi_{1}=12-4=8$.

Next, to find $\xi_{2}$, we take $\xi_{1}-\left(\pi_{1}-\pi_{2}\right)+P\left(\pi_{1}+\pi_{2}\right)=8-(13-8)+1=4$. Thus $\xi_{3}=4$ also.

To find $\xi_{4}$, we take $\xi_{1}-\left(\pi_{1}-\pi_{2}+\pi_{3}-\pi_{4}\right)+P\left(\pi_{1}+\pi_{2}\right)+P\left(\pi_{3}+\pi_{4}\right)=8-(13-8+$ $7-5)+1+0=2$. Thus $\xi_{5}=2$, also.

Similarly, we find $\xi_{6}=\xi_{7}=2$ and $\xi_{i}=0$ for $i \geqq 8$. Thus we have $\xi=(8,4,4,2,2,2,2)$ and from this we immediately get $\zeta=\left\langle 1^{4} 2^{6} 3^{5} 45\right\rangle$ by subtracting $\pi_{i}-\xi_{i}$ for $i \geqq 1$.

Having gotten this far, the rest is simple. $\lambda=(5+4,3+3,3+3,3+2,2+2,2+2,2+$ $1,1+1,1+0)=(9,6,6,5,4,4,3,2,1)$, and $\mu=\xi^{\prime}=(7,7,3,3,1,1,1,1)$. Finally, $\kappa=\lambda \cup \mu=$ $(9,7,7,6,6,5,4,4,3,3,3,2,1,1,1,1,1)$.

Notes. (i) I have written a Maple package which performs all of the mappings described in this paper, and also provides additional examples (http://www.math.rutgers. edu/ asills/papers.html).

(ii) It has come to my attention that Boulet [3] and Yee [7] have independently discovered additional combinatorial proofs of Theorem 1.1.

ACKNOWLEDGMENTS. I am grateful to George Andrews for initially supplying the problem and to the referees for their many helpful suggestions. I also wish to express my thanks to the organizers of the Baton Rouge $q$-series special session, Mourad E. H. Ismail and Stephen C. Milne, for the opportunity to present this material. This paper provides the full account of the original presentation given in the special session on $q$-series at the AMS Sectional Meeting in Baton Rouge on March 15, 2003 [5].

\section{REFERENCES}

[1] G. E. Andrews, The Theory of Partitions, Encyclopedia of Mathematics and Its Applications, vol. 2, Addison-Wesley, Massachusetts, 1976.

[2] - On a partition function of Richard Stanley, 11 (2004), no. 2, R1, Electronic J. Combin.

[3] C. Boulet, A four-parameter partition identity, to appear in Ramanujan J.

[4] I. G. Macdonald, Symmetric Functions and Hall Polynomials, 2nd ed., Oxford Mathematical Monographs, The Clarendon Press, Oxford University Press, New York, 1995.

[5] A. V. Sills, A combinatorial proof of a partition identity of Andrews and Stanley, Special Session on $Q$-Series in Number Theory and Combinatorics, AMS Sectional Meeting no. 984, Louisiana, March 2003, AMS Abstract 984-05-102.

[6] R. P. Stanley, Problem 10969, American Mathematical Monthly 109 (2002), 760.

[7] A. J. Yee, On partition functions of Andrews and Stanley, to appear in J. Combin. Theory Ser. A.

Andrew V. Sills: Department of Mathematics, Rutgers University, Piscataway, NJ 08854-8019, USA

E-mail address: asi11s@math.rutgers.edu 


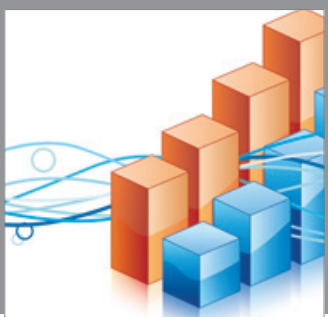

Advances in

Operations Research

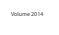

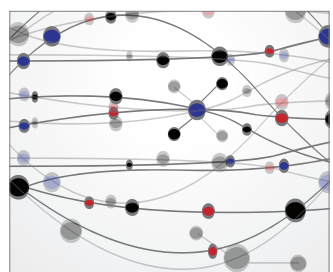

\section{The Scientific} World Journal
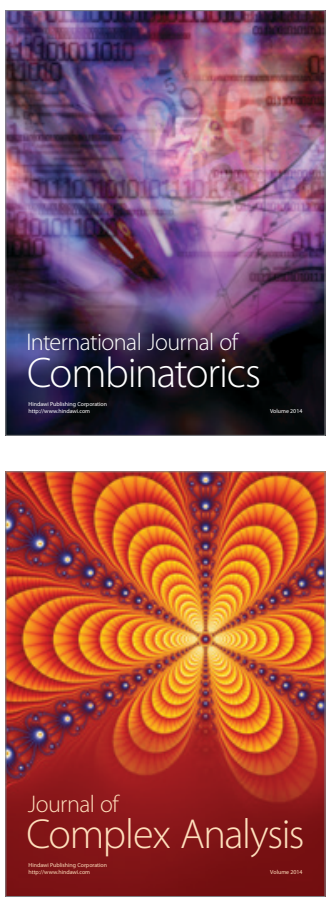

International Journal of

Mathematics and

Mathematical

Sciences
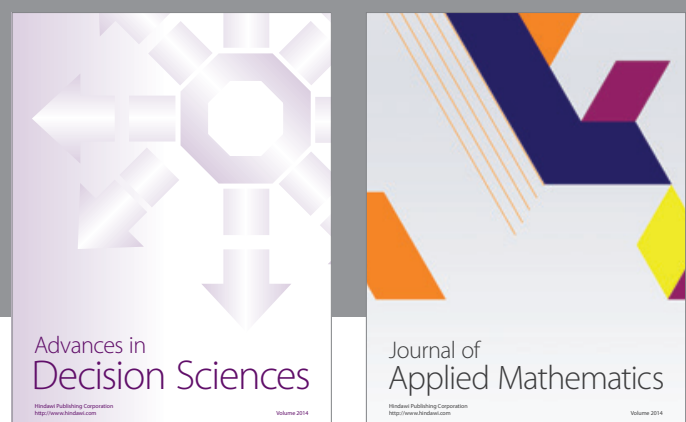

Journal of

Applied Mathematics
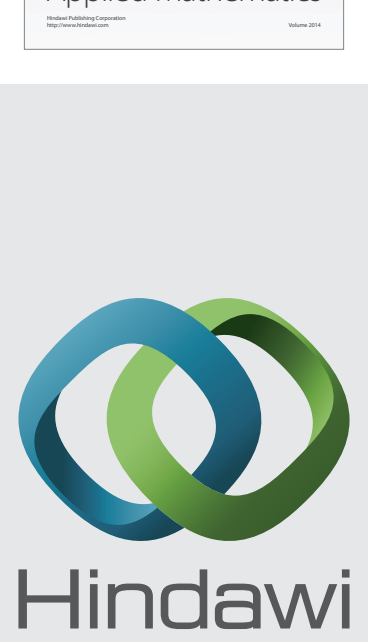

Submit your manuscripts at http://www.hindawi.com
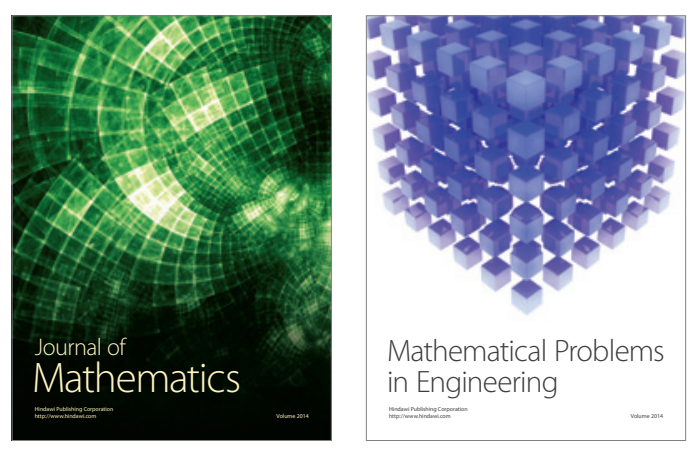

Mathematical Problems in Engineering
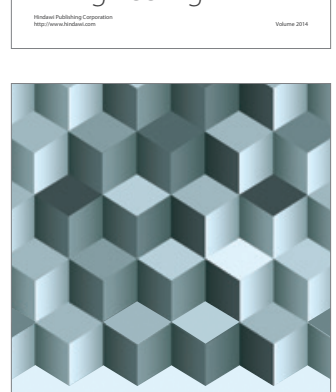

Journal of

Function Spaces
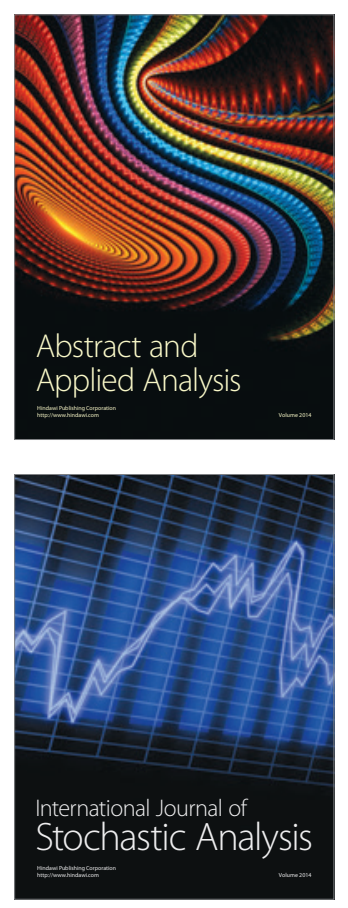

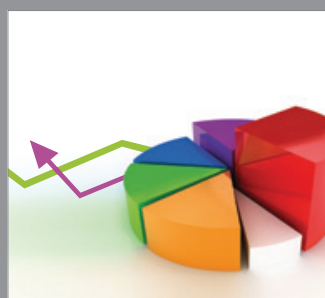

ournal of

Probability and Statistics

Promensencen
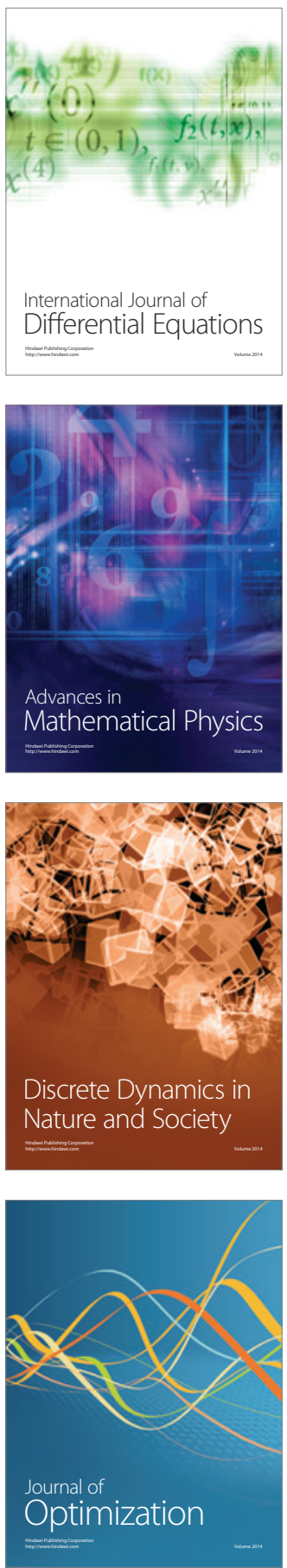